\title{
Dietary patterns and cognitive function risk in the elderly in a county of Guangxi: a cross-sectional study
}

Ruoyu Gou ( $\sim$ GRYgouruoyu@163.com )

Guilin Medical University

You Li

Guilin Medical University

Weiyi Pang

Guilin Medical University

Chunbao Mo

Guilin Medical University

Jiansheng Cai

Guangxi Medical University

Tingyu Luo

Guilin Medical University

Tingyu Mai

Guilin Medical University

Dechan Tan

Guilin Medical University

Kailian He

Guilin Medical University

Song Xiao

Guilin Medical University

Xu Tang

Guangxi Medical University

Hongnan Li

Guilin Medical University

Zhiyong Zhang

Guilin Medical University

Jian Qin

Guangxi Medical University 
Keywords: cognitive function, elderly population, oil tea, traditional diet, balanced diet

Posted Date: October 1st, 2021

DOI: https://doi.org/10.21203/rs.3.rs-944450/v2

License: (c) (1) This work is licensed under a Creative Commons Attribution 4.0 International License. Read Full License 


\section{Abstract}

Objective: To explore the effects of changes in dietary patterns on the cognitive functions of elderly people aged 60 and above in Gongcheng County.

Methods: A cross-sectional survey was conducted to study the health status of the elderly population in Gongcheng County, Guangxi. A quantitative food frequency table was used in obtaining information about eating habits, and the Chinese version of the Simple Mental State Examination Scale was used in obtaining the cognitive function score. Three main dietary patterns were obtained through factor analysis, and the significance of the main dietary structure and cognitive function was analyzed through logistic regression.

Results: This study covered 1246 elderly patients, of which 221 had cognitive impairments, accounting for $17.7 \%$. Three dietary patterns were extracted. The cereal and potato dietary model and oil tea-type dietary model had no protective effects on cognitive function $(P>0.05)$, whereas the vegetable and fruitbased diet pattern exerted a protective effect on cognition before and after the adjustment of potential confounding factors. This protective effect alleviated decline in cognitive function (before adjustment for confounding factors: odds ratio $[\mathrm{OR}]=2.05 ; 95 \%$ confidence interval $[\mathrm{Cl}]=1.34-3.15 ; \mathrm{P}<0.05$; after adjustment, $\mathrm{OR}=2.11,95 \% \mathrm{Cl}=1.34-3.33$, and $\mathrm{P}<0.05)$.

Conclusion: Traditional dietary patterns: (grain and potato dietary models and oil-tea-type dietary patterns) cannot alleviate cognitive decline. This study suggests that a specific structure of dietary habits (vegetable and fruit-based dietary patterns) can protect the elderly against cognitive decline.

\section{Introduction}

As the global aging situation is becoming more and more serious, senile diseases have received considerable attention[1], including chronic diseases, physical function, cognitive ability, and more than 10 middle-aged and elderly diseases[2]. Cognitive impairment has many causes, and Alzheimer's disease is the most common[3]. More than 46 million people are living with dementia worldwide. This number is expected to increase to 131.5 million by 2050[4]. China's population is aging[5]. In 2020, the prevalence of cognitive decline in China is $15.54 \%$, and the incidence rate of dementia is $6.04 \%$ [6]. Progressive memory loss, decline in language skills, and cognitive dysfunction in other areas have gradually reduced the elderly's ability to live independently and safely and increased their risk of having personality changes and intellectual loss. Therefore, cognitive dysfunction is in a precarious situation in the global health field. A variety of factors lead to the occurrence of dementia, such as age, apolipoprotein E genotype c4 allele, family history of dementia, living habits, and trauma[7,8]. Cognitive aging is not static[9], and interventions can be used to prevent the progression of the disease, providing evidence that diet intervention prevents the occurrence and development of dementia[10-13]. On the one hand, on the basis of the impacts of single components and nutritional supplements on cognition, increased intake of fish[14] and omega-3 polyunsaturated fatty acids and high linoleic acid[15-17]can prevent cognitive 
decline. On the other hand, nutritional intake has a synergistic effect on cognitive function[18,19]. Adherence to Mediterranean diet [20-24] and neurodegenerative delay (MIND) diet may delay cognitive decline[25,26]. Extracting dietary patterns are useful in exploring the mechanism of diet's influence on cognitive function, and factor analysis is a generally recognized mathematical model for exploring cognitive disorders and specific dietary patterns[3]. In summary, this study used factor analysis to explore the influence of Gongcheng County's dietary patterns on cognitive function.

\section{Materials And Methods}

\subsection{Data sources}

All research subjects in this study were selected from the residents of a county in Guangxi Zhuang Autonomous Region. According to the principle of cluster random sampling, 4356 residents aged 30 and above were selected from the villages and towns in the district, household questionnaires were used, and intensive health examinations and blood sampling were performed. Samples were tested for biochemical indicators. The inclusion criteria were as follows: permanent residents who were 60 years old or older at the time of the survey. Exclusion criteria: (1) people with mental illness; (2) people with incomplete physical examination and questionnaire data; (3) people with total energy intake greater than $6000 \mathrm{kcal}$ or less than $500 \mathrm{kcal}[27,28]$. After the implementation of the exclusion criteria, a total of 1246 adults over 60 years old were included in this study, comprising 531 males and 715 females. The ethics was reviewed and approved by the ethics committee of the School of Medicine of Guilin Medical College, and informed consent forms were signed for the participation system.

\subsection{Assessment of cognitive function}

The Mental State Examination Scale (MMSE) questionnaire has high reliability and validity in the evaluation of cognitive function and has certain advantages. It can be easily applied and requires a short time to implement[29]. MMSE scores are divided according to the level of education: illiterate group with $\leq 17$ points, elementary school group with $\leq 20$ points, and middle school and above groups with $\leq 24$ points were included in the cognitive impairment group[30], and the rest were included in the cognitively normal group.

\subsection{Dietary pattern study design}

The food frequency survey method and the food frequency survey table[31] combined with the local market sales in Gongcheng were used in determining the types of food and main food consumed by the research subjects in the past year, including the frequency and quantity of food intake, to reflect the intake of nutrients of the research subjects over a long period of time[32].

\subsection{Definition of terms}

Abnormal blood glucose is defined as follows: $F B G \geq 7.0 \mathrm{mmol} / \mathrm{L}[33]$ or taking hypoglycemic drugs. Hypertension is defined as systolic blood pressure of $\geq 140 \mathrm{mmHg}$ and/or diastolic blood pressure $\geq 90$ 
$\mathrm{mmHg}$ or taking antihypertensive drugs[34]. Dyslipidemia is defined as TC of $\geq 6.22 \mathrm{mmol} / \mathrm{L}, \mathrm{TG}$ of $\geq 2.26 \mathrm{mmol} / \mathrm{L}, \mathrm{LDL}-\mathrm{C}$ of $\geq 4.14 \mathrm{mmol} / \mathrm{L}$ for high LDL-C, and HDL-C of $<1.04 \mathrm{mmol} / \mathrm{L}$ for low HDL-C[35]. Body mass index is divided into three groups: $<18.5 \mathrm{~kg} / \mathrm{m}^{2}, 18.5 \sim 24.9 \mathrm{~kg} / \mathrm{m}^{2}$, and $25.0 \sim 30.0 \mathrm{~kg} / \mathrm{m}^{2}[36]$.

2.5.You Cha is the main component of the You Cha diet

As a drink, "oil tea" is largely consumed by people in the northeast of Guangxi, and some people consume oil tea three times a day during meals. Gongcheng oil tea, the most well-known oil tea produced from Guangxi Province, is produced with local tea, ginger, and, edible oil. The process involves soaking tea with warm water and heating when the tea bubble is soft. Ginger is ground with a "7 character" tea hammer, edible oil is added, and salt is added to taste. Tea is placed and hot water is added and then boiled[37].

\subsection{Statistical methods}

According to the similarity of food types and nutrition, 109 food products in the FFQ questionnaire were divided into 14 groups. The factor analysis method was used in solving the collinearity of the components, VARIMAX rotation was used in extracting the factor load value, the diet mode was determined, and the diet mode was named according to the factor load: vegetable-fruit type dietary pattern (high intake of vegetables and fruits and low intake of eggs, soy nuts, meat and alcohol), potato diet model (more cereals and potato-based food are consumed, and rice is the main products in Gongcheng County, Guangxi, China), and oil tea-type diet model (high intake of oil tea as obvious signs), as shown in Table. 1. Participants' factor scores are grouped according to quartiles. The number of dietary patterns is determined with eigenvalues[38]. The Kaiser-Meyer-Olkin Measure of Sampling Adequacy value was 0.791 , Bartlett's Test of Sphericity was less than or equal to 0.001 , the cumulative variance explanation rate was $82.02 \%$, and the matrix standard was 0.5 . Dietary patterns with factor load values larger than this standard indicate that they were extremely related to this pattern and were named accordingly.SPSS 21.0 was used in statistical analysis, and linear analysis of continuous variables and chi-square test of categorical variables were used in obtaining linear trends between the quartiles of dietary pattern scores. The groups were compared using $t$ test or analysis of variance, and the nonnormally distributed data groups were compared with Wilcoxon test. After adjustment for potential confounding factors, regression of binary logistic was used in analyzing the influencing factors of the cognitive function status of the elderly, and a P value of $<0.05$ was considered statistically significant. 
Table. 1 Rotated factor loading matrix for dietary patterns

\begin{tabular}{cccc}
\hline Food group & Vegetables and fruits & Cereals and potatoes & You Cha \\
\hline Vegetables & $\mathbf{0 . 8 2 8}^{\mathrm{a}}$ & 0.137 & - \\
\hline Fruits & $\mathbf{0 . 8 1 8 ^ { \mathrm { a } }}$ & 0.125 & - \\
\hline Soy Nuts & 0.393 & 0.152 & - \\
\hline Eggs & 0.286 & - & - \\
\hline Meats & 0.274 & 0.102 & - \\
\hline Fungi food & 0.265 & 0.100 & - \\
\hline Aquatic & 0.249 & - & - \\
\hline Edible viscera & 0.170 & 0.148 & - \\
\hline Dairy & 0.124 & - & - \\
\hline Alcohol product & 0.112 & - & - \\
\hline Salt & - & - & - \\
\hline Edible oil & - & - & $0.998^{\mathrm{a}}$ \\
\hline Cereals and potatoes & 0.127 & - & \\
\hline You Cha & - & & $-991^{\mathrm{a}}$ \\
\hline
\end{tabular}

Absolute values $\square 0.5$ are not listed for simplicity

${ }^{\mathrm{a}}$ Absolute values $\square 0.5$

\section{Results}

3.1General characteristics of the research object

In this survey, CL male patients were fewer than females. Men and women aged 60-69 accounted for the majority, accounting for $73.6 \%$ and $74.0 \%$, respectively. Among the participants, $68.0 \%$ were Yao men, $63.2 \%$ were women, $81.2 \%$ were married, and $81.2 \%$ were men. With regard to other conditions (such as divorce, separation, and death), men were not dominant, women were more illiterate, and men suffered. The education levels of men were higher than those of women. The occupation was mainly farming. Other occupations (such as officials, businessmen, and migrant workers) were dominated by males, which accounted for $8.3 \%$. The proportion of men suffering from chronic diseases was $64.4 \%$, and the proportion of women was $65.7 \%$. The smoking and drinking rates of men were significantly higher than those of women. However, the proportion of women exposed to second-hand smoke was $61.7 \%$, which was higher than that of men. The body mass index ranged from $18.5 \mathrm{~kg} / \mathrm{m}^{2}$ to $24.9 \mathrm{~kg} / \mathrm{m}^{2}$, and the proportion of men was $70.8 \%$ more than that of women. The average daily walking and sitting time were $2.9 \pm 2.1$ and $3.6 \pm 1.82 \mathrm{~h}$, respectively, as shown in Table. 2 and Table.3. 
Table.2 Comparison of cognitive function status in elderly people of different genders

\begin{tabular}{|c|c|c|c|c|}
\hline & CL口 \% & Normal & $X^{2}$ & $\mathrm{P}$ \\
\hline Man & 71口13.4ロ & 460ロ86.6ロ & 12.088 & 0.001 \\
\hline Women & $150(21.0)$ & $565 \square 79.0 \square$ & & \\
\hline Total & 221ロ17.7ロ & $1025 \square 82.3 \square$ & & \\
\hline
\end{tabular}


Table. 3 General characteristics of the study subjects

\begin{tabular}{|c|c|c|c|c|}
\hline & $\operatorname{Man} \square \% \square$ & Women & $x^{2}$ & $\mathrm{P}^{\mathrm{a}}$ \\
\hline \multicolumn{5}{|l|}{ Age(years) } \\
\hline$\geq 60$ & 391 (73.6) & $529(74)$ & 0.073 & 0.964 \\
\hline$\geq 70$ & $118(22.2)$ & $155(21.7)$ & & \\
\hline$\geq 80$ & $22(4.1)$ & $31(4.3)$ & & \\
\hline \multicolumn{5}{|l|}{ Ethnic } \\
\hline ethnic Han & $152(28.6)$ & $231(32.3)$ & 3.301 & 0.192 \\
\hline ethnic Yao & 361 (68) & $452(63.2)$ & & \\
\hline Rest & $18(3.4)$ & $32(4.5)$ & & \\
\hline \multicolumn{5}{|l|}{ Marital status } \\
\hline spinsterhood & $28(5.3)$ & $3(0.4)$ & 59.839 & 0.000 \\
\hline married & $431(81.2)$ & $515(72.0)$ & & \\
\hline Rest & $72(13.6)$ & $197(27.6)$ & & \\
\hline \multicolumn{5}{|l|}{ Education } \\
\hline Illiteracy & $189(35.6)$ & $474(66.3)$ & 128.890 & 0.000 \\
\hline primary & $133(25.0)$ & $131(18.3)$ & & \\
\hline Above education & 209 (39.4) & $110(15.4)$ & & \\
\hline \multicolumn{5}{|l|}{ Career } \\
\hline Farmer & 487 (91.7) & $687(96.1)$ & 10.688 & 0.001 \\
\hline Reest & $44(8.3)$ & $28(3.9)$ & & \\
\hline \multicolumn{5}{|l|}{ Income } \\
\hline$\leq 2000$ & $86(16.2)$ & $155(21.7)$ & 7.799 & 0.020 \\
\hline $2000 \square 5000$ & $82(15.4)$ & $123(17.2)$ & & \\
\hline$\geq 5000$ & $363(68.4)$ & $437(61.1)$ & & \\
\hline \multicolumn{5}{|l|}{ Disease $^{d}$} \\
\hline Not & $189(35.6)$ & $245(34.3)$ & 0.237 & 0.627 \\
\hline Yes & $342(64.4)$ & $470(65.7)$ & & \\
\hline \multicolumn{5}{|l|}{ Smoke } \\
\hline Not & $288(54.2)$ & 707 (98.9) & 377.524 & 0.000 \\
\hline Yes & $243(45.8)$ & $8(1.1)$ & & \\
\hline \multicolumn{5}{|l|}{ Passive smoking } \\
\hline Not & $298(56.1)$ & $274(38.3)$ & 0.389 & 0.000 \\
\hline Yes & 233 (43.9) & 441 (61.7) & & \\
\hline \multicolumn{5}{|l|}{ Drink } \\
\hline Not & $250(47.1)$ & $560(78.3)$ & 130.734 & 0.000 \\
\hline Yes & 281 (52.9) & $155(21.7)$ & & \\
\hline \multicolumn{5}{|l|}{ SBP } \\
\hline Abnormal & $248(46.7)$ & 367 (51.3) & 2.607 & 0.106 \\
\hline Normal & $283(53.3)$ & 348 (48.7) & & \\
\hline \multicolumn{5}{|l|}{ DBP } \\
\hline Abnormal & $139(26.2)$ & $227(31.7)$ & 4.559 & 0.033 \\
\hline Normal & $392(73.8)$ & $488(68.3)$ & & \\
\hline \multicolumn{5}{|l|}{ Blood glucose } \\
\hline Abnormal & $29(5.5)$ & $35(4.9)$ & 0.201 & 0.654 \\
\hline Normal & $502(94.5)$ & $680(95.1)$ & & \\
\hline \multicolumn{5}{|l|}{ BMI } \\
\hline$<18.5 \mathrm{~kg} / \mathrm{m}^{2}$ & $37(7.0)$ & 85 (11.9) & 9.317 & 0.009 \\
\hline
\end{tabular}




\begin{tabular}{ccccc}
\hline $18.5 \sim 24.9 \mathrm{~kg} / \mathrm{m}^{2}$ & $376(70.8)$ & $495(69.2)$ & & \\
\hline $25.0 \sim 30.0 \mathrm{~kg} / \mathrm{m}^{2}$ & $118(22.2)$ & $135(18.9)$ & & \\
\hline LDL & & & & \\
\hline Abnormal & $125(23.5)$ & $203(28.4)$ & 3.697 & 0.055 \\
\hline Normal & $406(76.5)$ & $512(71.6)$ & & \\
\hline TG & $57(10.7)$ & $75(10.5)$ & 0.019 & 0.890 \\
\hline Abnormal & $474(89.3)$ & $640(89.5)$ & & \\
\hline Normal & $7(1.3)$ & $2(0.3)$ & 4.583 & 0.032 \\
\hline HDL-C & $524(98.7)$ & $713(99.7)$ & & \\
\hline Abnormal & $5.53 \pm 1.11$ & $5.74 \pm 1.16$ & 0.032 & 0.859 \\
\hline Normal & $87.38 \pm 12.5$ & $87.19 \pm 7.87$ & 6.605 & 0.010 \\
\hline TC & $78.55 \pm 13.28$ & $76.89 \pm 10.28$ & 2.693 & 0.101 \\
\hline Hipline & $2.9 \pm 2.1$ & $2.95 \pm 2.13$ & 0.582 & 0.446 \\
\hline Waist & $3.6 \pm 1.82$ & $3.56 \pm 1.82$ & 0.002 & 0.964 \\
\hline Walking Time & & & \\
\hline Meditation time & & & \\
\hline${ }^{\mathrm{a} T}$ test for continuous variables and Chi-square test for categorical variables \\
\hline${ }^{\mathrm{b}} \mathrm{n}$ ( \%) \\
\hline${ }^{\mathrm{c}}$ Mean \pm SD \\
\hline${ }^{\mathrm{d}}$ Presence of disease (hypertension, diabetes, cardiovascular disease, or stroke)
\end{tabular}

\subsection{MMSE of experimental and control groups in different genders}

The cognitive impairment group of different genders had a lower total MMSE score than the control group and lower scores for the five cognitive function assessment indicators of orientation, attention, calculation, memory, language ability, and visual space than the normal group (Pख0 001). The median total score of cognitive function in the male normal group was 27 points, and the cognitive impairment group had a score of 20 points. The scores of various items and total scores of male cognitive function were higher than those of the female group, as shown in Table.4.

[See the supplementary files section to view Table 4.]

3.3.Baseline characteristics of subjects in the lowest (Q1) and highest (Q4) quartile of each model

The three dietary patterns were determined, and the confounding variables were adjusted. The higher the score of the vegetable and fruit diet model was, the lower the proportion of farmers engaged in occupations, the better the marital status, the higher the education level, and the higher the number of high-income groups were. The proportion of chronic diseases showed a decreasing trend, the proportion of people without chronic diseases increased, the number of people whose diastolic blood pressure levels 
were in the normal range increased, the body mass index increased, the average daily walking time decreased to $2.7 \pm 1.9 \mathrm{~h}$, and the rest time increased to $3.8 \pm 1.8 \mathrm{~h}$. In the dietary pattern of cereals and potatoes, the blood sugar level showed a decreasing trend. The number of smokers in the oil tea-type dietary pattern increased, and the diastolic blood pressure tended to increase as a whole, as shown in Table.5. The variables showing a significant trend in the quartile of dietary pattern scores were screened and then entered into the model and adjusted as potential confounding factors. 
Table. 5 Characteristics of the subjects in the lowest (Q1) and highest (Q4) quartiles of each pattern

\begin{tabular}{|c|c|c|c|c|c|c|c|c|c|}
\hline & \multicolumn{2}{|c|}{ Vegetables and fruits } & \multirow[b]{2}{*}{$\mathrm{P}^{\mathrm{b}}$} & \multicolumn{2}{|c|}{$\begin{array}{c}\text { Cereals and } \\
\text { potatoes }\end{array}$} & \multicolumn{3}{|c|}{ You Cha } & \multirow[b]{2}{*}{$\mathrm{P}$} \\
\hline & $\mathrm{Q} 1^{\mathrm{a}}$ & Q4 & & Q1 & Q4 & $\mathrm{P}$ & Q1 & Q4 & \\
\hline $\mathrm{n}$ & 312 & 311 & & 311 & 311 & & 312 & 312 & \\
\hline \multicolumn{10}{|l|}{ Sex } \\
\hline $\operatorname{Man} \square \% \square$ & 116(37.1) & $\begin{array}{c}153 \\
(49.1)\end{array}$ & \multirow[t]{2}{*}{0.002} & $\begin{array}{c}114 \\
(36.6)\end{array}$ & $\begin{array}{c}150 \\
(48.2)\end{array}$ & \multirow[t]{2}{*}{0.003} & $125(40)$ & $\begin{array}{c}167 \\
(53.5)\end{array}$ & \multirow[t]{2}{*}{0.001} \\
\hline Women & 196(62.8) & $\begin{array}{c}158 \\
(50.8)\end{array}$ & & $\begin{array}{c}197 \\
(63.3)\end{array}$ & $\begin{array}{c}161 \\
(51.7)\end{array}$ & & $\begin{array}{c}187 \\
(59.9)\end{array}$ & $\begin{array}{c}145 \\
(46.4)\end{array}$ & \\
\hline \multicolumn{10}{|l|}{ Age(years) } \\
\hline$\geq 60$ & $230(73.7)$ & $\begin{array}{c}241 \\
(77.4)\end{array}$ & \multirow[t]{3}{*}{0.378} & $\begin{array}{c}234 \\
(75.2)\end{array}$ & $\begin{array}{c}229 \\
(73.6)\end{array}$ & \multirow[t]{3}{*}{0.474} & $\begin{array}{c}230 \\
(73.7)\end{array}$ & $\begin{array}{c}241 \\
(77.2)\end{array}$ & \multirow[t]{3}{*}{0.214} \\
\hline$\geq 70$ & $72(23)$ & $58(18.6)$ & & $64(20.5)$ & $64(20.5)$ & & $63(20.1)$ & $61(19.5)$ & \\
\hline$\geq 80$ & $10(3.2)$ & $12(3.8)$ & & $13(4.1)$ & $18(5.7)$ & & $19(6)$ & $10(3.2)$ & \\
\hline \multicolumn{10}{|l|}{ Ethnic } \\
\hline ethnic Han & 108(34.6) & $85(27.3)$ & \multirow[t]{3}{*}{0.088} & $85(27.3)$ & $97(31.1)$ & \multirow[t]{3}{*}{0.396} & 99 (31.7) & $97(31)$ & \multirow[t]{3}{*}{0.210} \\
\hline ethnic Yao & $195(62.5)$ & $\begin{array}{c}211 \\
(67.8)\end{array}$ & & $\begin{array}{c}214 \\
(68.8)\end{array}$ & $\begin{array}{c}201 \\
(64.6)\end{array}$ & & $\begin{array}{c}194 \\
(62.1)\end{array}$ & $\begin{array}{c}205 \\
(65.7)\end{array}$ & \\
\hline Rest & $9(2.8)$ & $15(4.8)$ & & $12(3.8)$ & $13(4.1)$ & & $19(6)$ & $10(3.2)$ & \\
\hline \multicolumn{10}{|l|}{ Marital status } \\
\hline spinsterhood & $13(4.1)$ & $2(0.6)$ & \multirow[t]{3}{*}{0.000} & $8(2.5)$ & $9(2.8)$ & \multirow[t]{3}{*}{0.931} & $6(1.9)$ & $8(2.5)$ & \multirow[t]{3}{*}{0.784} \\
\hline married & $213(68.2)$ & $\begin{array}{c}262 \\
(84.2)\end{array}$ & & $\begin{array}{c}234 \\
(75.2)\end{array}$ & $\begin{array}{c}231 \\
(74.2)\end{array}$ & & $\begin{array}{c}235 \\
(75.3)\end{array}$ & $\begin{array}{c}238 \\
(76.2)\end{array}$ & \\
\hline Rest & $86(27.5)$ & $47(15.1)$ & & $69(22.1)$ & $71(22.8)$ & & 71 (22.7) & $66(21.1)$ & \\
\hline \multicolumn{10}{|l|}{ Education } \\
\hline Illiteracy & $202(64.7)$ & $\begin{array}{c}116 \\
(37.2)\end{array}$ & \multirow[t]{3}{*}{$0.000^{\mathrm{d}}$} & $\begin{array}{c}173 \\
(55.6)\end{array}$ & $\begin{array}{c}153 \\
(49.1)\end{array}$ & \multirow[t]{3}{*}{0.057} & $\begin{array}{c}154 \\
(49.3)\end{array}$ & $\begin{array}{c}154 \\
(49.3)\end{array}$ & \multirow[t]{3}{*}{0.994} \\
\hline primary & $65(20.8)$ & $76(24.4)$ & & 68 (21.8) & $68(21.8)$ & & $65(20.8)$ & $66(21.1)$ & \\
\hline $\begin{array}{c}\text { Above } \\
\text { education }\end{array}$ & $45(14.4)$ & $\begin{array}{c}119 \\
(38.2)\end{array}$ & & $70(22.5)$ & 90 (28.9) & & $93(29.8)$ & $92(29.4)$ & \\
\hline \multicolumn{10}{|l|}{ Career } \\
\hline Farmer & $303(97.1)$ & $\begin{array}{c}287 \\
(92.2)\end{array}$ & \multirow[t]{2}{*}{0.007} & $\begin{array}{c}294 \\
(94.5)\end{array}$ & $\begin{array}{c}294 \\
(94.5)\end{array}$ & 1.000 & $\begin{array}{c}293 \\
(93.9)\end{array}$ & $\begin{array}{c}296 \\
(94.8)\end{array}$ & 0.602 \\
\hline Reest & $9(2.8)$ & $24(7.7)$ & & $17(5.4)$ & $17(5.4)$ & & $19(6)$ & $16(5.1)$ & \\
\hline Income & & & & & & & & & \\
\hline$\leq 2000$ & $60(19.2)$ & 58 (18.6) & 0.008 & 59 (18.9) & 55 (17.6) & 0.683 & $60(19.2)$ & 62 (19.8) & 0.821 \\
\hline $2000 \square 5000$ & $67(21.4)$ & 39 (12.5) & & 55 (17.6) & 55 (17.6) & & 59 (18.9) & 53 (16.9) & \\
\hline$\geq 5000$ & $185(59.2)$ & $\begin{array}{c}214 \\
(68.8)\end{array}$ & & $\begin{array}{c}197 \\
(63.3)\end{array}$ & $\begin{array}{c}201 \\
(64.6)\end{array}$ & & $\begin{array}{c}193 \\
(61.8)\end{array}$ & $\begin{array}{c}197 \\
(63.1)\end{array}$ & \\
\hline Disease $^{\mathrm{e}}$ & & & & & & & & & \\
\hline Not & $91(29.1)$ & $\begin{array}{c}121 \\
(38.9)\end{array}$ & 0.010 & $99(31.8)$ & $\begin{array}{c}121 \\
(38.9)\end{array}$ & 0.065 & $\begin{array}{c}102 \\
(32.6)\end{array}$ & $\begin{array}{c}117 \\
(37.5)\end{array}$ & 0.208 \\
\hline Yes & $221(70.8)$ & $190(61)$ & & $\begin{array}{c}212 \\
(68.1)\end{array}$ & $190(61)$ & & $\begin{array}{c}210 \\
(67.3)\end{array}$ & $\begin{array}{c}195 \\
(62.5)\end{array}$ & \\
\hline Medicine $^{f}$ & & & & & & & & & \\
\hline Not & $129(41.3)$ & $\begin{array}{c}150 \\
(48.2)\end{array}$ & 0.084 & $\begin{array}{c}132 \\
(42.4)\end{array}$ & $\begin{array}{c}156 \\
(50.1)\end{array}$ & 0.054 & $\begin{array}{c}131 \\
(41.9)\end{array}$ & $\begin{array}{c}147 \\
(47.1)\end{array}$ & 0.198 \\
\hline
\end{tabular}




\begin{tabular}{|c|c|c|c|c|c|c|c|c|c|}
\hline Yes & $183(58.6)$ & $\begin{array}{c}161 \\
(51.7)\end{array}$ & & $\begin{array}{c}179 \\
(57.5)\end{array}$ & $\begin{array}{c}155 \\
(49.8)\end{array}$ & & $181(58)$ & $\begin{array}{c}165 \\
(52.8)\end{array}$ & \\
\hline \multicolumn{10}{|c|}{ Smoke } \\
\hline Not & $253(81)$ & $\begin{array}{c}237 \\
(76.2)\end{array}$ & 0.137 & $\begin{array}{c}261 \\
(83.9)\end{array}$ & $\begin{array}{c}244 \\
(78.4)\end{array}$ & 0.081 & $256(82)$ & $\begin{array}{c}227 \\
(72.7)\end{array}$ & 0.006 \\
\hline Yes & 59 (18.9) & $74(23.7)$ & & $50(16)$ & $67(21.5)$ & & $56(17.9)$ & $85(27.2)$ & \\
\hline
\end{tabular}




\begin{tabular}{|c|c|c|c|c|c|c|c|c|c|}
\hline $\begin{array}{l}\text { Passive } \\
\text { smoking }\end{array}$ & & & \multirow{3}{*}{0.159} & & & \multirow{3}{*}{0.520} & & & \multirow{3}{*}{0.378} \\
\hline Not & $154(49.3)$ & $\begin{array}{c}136 \\
(43.7)\end{array}$ & & $\begin{array}{c}139 \\
(44.6)\end{array}$ & $\begin{array}{c}147 \\
(47.2)\end{array}$ & & $\begin{array}{c}152 \\
(48.7)\end{array}$ & $\begin{array}{c}141 \\
(45.1)\end{array}$ & \\
\hline Yes & $158(50.6)$ & $\begin{array}{c}175 \\
(56.2)\end{array}$ & & $\begin{array}{c}172 \\
(55.3)\end{array}$ & $\begin{array}{c}164 \\
(52.7)\end{array}$ & & $\begin{array}{c}160 \\
(51.2)\end{array}$ & $\begin{array}{c}171 \\
(54.8)\end{array}$ & \\
\hline \multicolumn{10}{|l|}{ Drink } \\
\hline Not & $216(69.2)$ & $\begin{array}{c}201 \\
(64.6)\end{array}$ & \multirow[t]{2}{*}{0.222} & $\begin{array}{c}215 \\
(69.1)\end{array}$ & $\begin{array}{c}194 \\
(62.3)\end{array}$ & \multirow[t]{2}{*}{0.076} & $\begin{array}{c}214 \\
(68.5)\end{array}$ & $\begin{array}{c}196 \\
(62.8)\end{array}$ & \multirow[t]{2}{*}{0.129} \\
\hline Yes & $96(30.7)$ & $\begin{array}{c}110 \\
(35.3)\end{array}$ & & $96(30.8)$ & $\begin{array}{c}117 \\
(37.6)\end{array}$ & & $98(31.4)$ & $\begin{array}{c}116 \\
(37.1)\end{array}$ & \\
\hline \multicolumn{10}{|l|}{ SBP } \\
\hline Abnormal & $167(53.5)$ & $\begin{array}{c}143 \\
(45.9)\end{array}$ & \multirow[t]{2}{*}{0.060} & $\begin{array}{c}142 \\
(45.6)\end{array}$ & $\begin{array}{c}153 \\
(49.1)\end{array}$ & \multirow[t]{2}{*}{0.377} & $\begin{array}{c}151 \\
(48.3)\end{array}$ & $\begin{array}{c}152 \\
(48.7)\end{array}$ & \multirow[t]{2}{*}{0.936} \\
\hline Normal & $145(46.4)$ & $168(54)$ & & $\begin{array}{c}169 \\
(54.3)\end{array}$ & $\begin{array}{c}158 \\
(50.8)\end{array}$ & & $\begin{array}{c}161 \\
(51.6)\end{array}$ & $\begin{array}{c}160 \\
(51.2)\end{array}$ & \\
\hline
\end{tabular}

DBP

\begin{tabular}{|c|c|c|c|c|c|c|c|c|c|}
\hline Abnormal & $107(34.2)$ & 82 (26.3) & 0.031 & 84 (27) & 93 (29.9) & 0.424 & $\begin{array}{c}102 \\
(32.6)\end{array}$ & 78 (25) & 0.034 \\
\hline Normal & $205(65.7)$ & $\begin{array}{c}229 \\
(73.6)\end{array}$ & & $\begin{array}{c}227 \\
(72.9)\end{array}$ & $218(70)$ & & $\begin{array}{c}210 \\
(67.3)\end{array}$ & $234(75)$ & \\
\hline
\end{tabular}

Blood

glucose

\begin{tabular}{|c|c|c|c|c|c|c|c|c|c|}
\hline Abnormal & $20(6.4)$ & $13(4.1)$ & \multirow[t]{2}{*}{0.214} & 31 (9.9) & 11 (3.5) & \multirow[t]{2}{*}{0.001} & $15(4.8)$ & $16(5.1)$ & \multirow[t]{2}{*}{0.854} \\
\hline Normal & 292(93.5) & $\begin{array}{c}298 \\
(95.8)\end{array}$ & & $280(90)$ & $\begin{array}{c}300 \\
(96.4)\end{array}$ & & $\begin{array}{c}297 \\
(95.1)\end{array}$ & $\begin{array}{c}296 \\
(94.8)\end{array}$ & \\
\hline \multicolumn{10}{|l|}{ BMI } \\
\hline$<18.5 \mathrm{~kg} / \mathrm{m} 2$ & $20(7.4)$ & 49 (13.8) & \multirow[t]{3}{*}{0.030} & $30(9.6)$ & 31 (9.9) & \multirow[t]{3}{*}{0.884} & 26 (8.3) & $26(8.3)$ & \multirow[t]{3}{*}{0.623} \\
\hline $\begin{array}{c}18.5 \sim 24.9 \\
\mathrm{~kg} / \mathrm{m} 2\end{array}$ & 188(69.8) & $\begin{array}{c}239 \\
(67.5)\end{array}$ & & $\begin{array}{c}212 \\
(68.1)\end{array}$ & $\begin{array}{c}212 \\
(68.1)\end{array}$ & & $\begin{array}{c}211 \\
(67.6)\end{array}$ & $\begin{array}{c}221 \\
(70.8)\end{array}$ & \\
\hline $\begin{array}{c}25.0 \sim 30.0 \\
\mathrm{~kg} / \mathrm{m} 2\end{array}$ & $61(22.6)$ & 66 (18.6) & & $69(22.1)$ & $68(21.8)$ & & $75(24)$ & $\begin{array}{c}65 \\
(20.8)\end{array}$ & \\
\hline \multicolumn{10}{|l|}{ LDL } \\
\hline Abnormal & $86(27.5)$ & $75(24.1)$ & \multirow[t]{2}{*}{0.326} & 74 (23.7) & $91(29.2)$ & \multirow[t]{2}{*}{0.123} & $77(24.6)$ & $\begin{array}{c}73 \\
(23.3)\end{array}$ & \multirow[t]{2}{*}{0.708} \\
\hline Normal & $226(72.4)$ & $\begin{array}{c}236 \\
(75.8)\end{array}$ & & $\begin{array}{c}237 \\
(76.2)\end{array}$ & $\begin{array}{c}220 \\
(70.7)\end{array}$ & & $\begin{array}{c}235 \\
(75.3)\end{array}$ & $\begin{array}{c}239 \\
(76.6)\end{array}$ & \\
\hline
\end{tabular}

TG

\begin{tabular}{|c|c|c|c|c|c|c|c|c|c|}
\hline Abnormal & 33 (10.5) & $30(9.6)$ & \multirow[t]{2}{*}{0.700} & 35 (11.2) & 29 (9.3) & \multirow[t]{2}{*}{0.428} & 36 (11.5) & 28 (8.9) & \multirow[t]{2}{*}{0.291} \\
\hline Normal & $279(89.4)$ & $\begin{array}{c}281 \\
(90.3)\end{array}$ & & $\begin{array}{c}276 \\
(88.7)\end{array}$ & $\begin{array}{c}282 \\
(90.6)\end{array}$ & & $\begin{array}{c}276 \\
(88.4)\end{array}$ & $284(91)$ & \\
\hline
\end{tabular}

HDL-C

\begin{tabular}{|c|c|c|c|c|c|c|c|c|c|}
\hline Abnormal & $1(0.3)$ & $5(1.6)$ & 0.100 & $2(0.6)$ & $2(0.6)$ & 1.000 & $3(0.9)$ & $1(0.3)$ & 0.316 \\
\hline Normal & $311(99.6)$ & $\begin{array}{c}306 \\
(98.3)\end{array}$ & & $\begin{array}{c}309 \\
(99.3)\end{array}$ & $\begin{array}{c}309 \\
(99.3)\end{array}$ & & 309 (99) & $\begin{array}{c}311 \\
(99.6)\end{array}$ & \\
\hline TC & $5.7 \pm 1.2^{\mathrm{C}}$ & $5.6 \pm 1.1$ & 0.429 & $5.7 \pm 1.1$ & $5.6 \pm 1.1$ & 0.844 & $5.6 \pm 1.2$ & $5.6 \pm 0.9$ & 0.243 \\
\hline Hipline & $86.4 \pm 11$ & $88.3 \pm 10.2$ & 0.329 & $87.7 \pm 10.1$ & $86.4 \pm 13.1$ & 0.695 & $87.9 \pm 12.6$ & $87.8 \pm 6.9$ & 0.461 \\
\hline Waist & $76.5 \pm 12.4$ & $78.2 \pm 12$ & 0.344 & $78.5 \pm 11.3$ & $76.7 \pm 13.8$ & 0.385 & $78.8 \pm 13.6$ & $78.1 \pm 9.3$ & 0.323 \\
\hline Walking & $2.9 \pm 2.1$ & $2.7 \pm 1.9$ & 0.049 & $\begin{array}{l}2.9 \pm 2.1 \\
\text { Page } 13\end{array}$ & $2.8 \pm 2.1$ & 0.529 & $2.7 \pm 2.1$ & $2.9 \pm 2.2$ & 0.544 \\
\hline
\end{tabular}


Time

$\begin{array}{llllllllll}\text { Meditation } & 3.5 \pm 1.7 & 3.8 \pm 1.8 & 0.034 & 3.7 \pm 1.7 & 3.5 \pm 1.9 & 0.088 & 3.6 \pm 1.9 & 3.6 \pm 1.9 & 0.921\end{array}$

time

BMI body mass index

${ }^{\mathrm{a}}$ Quartile

$\mathrm{b}_{\mathrm{P}}$ values for linear trend. General linear model for continuous variables and Chi-square test for categorical variables

$\mathrm{C}_{\text {Mean }} \pm \mathrm{SD}$

$\mathrm{d}_{\mathrm{P}}$ for the trend was calculated after educational level was divided by higher ,lower educational level and uneducated

${ }^{\mathrm{e}}$ Presence of disease (hypertension, diabetes, cardiovascular disease, or stroke)

$\mathrm{f}_{\text {Take the medicine within two weeks }}$

3.4.Nutrient characteristics of subjects in the lowest (Q1) and highest (Q4) quartile of each model

After adjustment according to age and gender, an obvious increasing trend associated with the intake of all nutrients, the vegetable and fruit-based diet pattern, and the cereal and potato diet model was observed (P区0.0001). However, in the cereal and potato diet model, increase in the intake of unsaturated fatty acids was nonsignificant $(P=0.0519)$. No significant difference in nutrient intake was found in the oil tea diet group (P凶0.05), as shown in Table.6.

[See the supplementary files section to view Table 6.]

3.5.Logistic regression analysis before and after adjustment for traditional dietary pattern and balanced vegetable and fruit-based dietary pattern

The relationship between eating patterns and cognitive impairment is shown in Table.7. In the cereal and potato diet and oil tea diet models, the initial model and the adjusted model were not related to cognitive impairment ( $P$ values were both greater than 0.05 ). When no adjustment for covariates was made (odds ratio $[\mathrm{OR}]$ of the fourth pair of first quantiles, $2.05 ; 95 \%$ confidence interval $[\mathrm{Cl}], 1.34-3.15 ; \mathrm{P}=0.000$ ). After the covariates were adjusted, in the vegetable and fruit diet (the OR of the fourth pair of the first quantile, $2.11 ; 95 \% \mathrm{Cl}, 1.34-3.33 ; \mathrm{P}=0.001$ ). 
Table. 7 Risk of cognitive impairment across quartiles of dietary pattern scores adjusted for covariates

\begin{tabular}{|c|c|c|c|c|c|c|}
\hline \multirow[t]{2}{*}{ Item } & \multicolumn{3}{|c|}{ Crude } & \multicolumn{3}{|c|}{ Model $^{\mathrm{a}}$} \\
\hline & $\mathrm{P}$ & OR & $95 \%$ CI & $\mathrm{P}$ & OR & $95 \% \mathrm{CI}$ \\
\hline \multicolumn{7}{|c|}{ vegetables and fruits } \\
\hline Q1 & 0.011 & & & 0.014 & & \\
\hline Q2 & 0.093 & 1.4 & $(0.94,2.08)$ & 0.103 & 1.39 & $(0.93,2.08)$ \\
\hline Q3 & 0.165 & 1.31 & $(0.89,1.94)$ & 0.197 & 1.3 & $(0.87,1.94)$ \\
\hline Q4 & 0.000 & 2.05 & $(1.34,3.15)$ & 0.001 & 2.11 & $(1.34,3.33)$ \\
\hline \multicolumn{7}{|c|}{ cereals and potatoes } \\
\hline Q1 & 0.803 & & & 0.804 & & \\
\hline Q2 & 0.566 & 1.12 & $(0.74,1.7)$ & 0.743 & 1.07 & $(0.7,1.62)$ \\
\hline Q3 & 0.424 & 1.18 & $(0.78,1.79)$ & 0.619 & 1.11 & $(0.73,1.69)$ \\
\hline Q4 & 0.999 & 0.99 & $(0.66,1.5)$ & 0.669 & 0.91 & $(0.6,1.37)$ \\
\hline \multicolumn{7}{|c|}{ You Cha } \\
\hline Q1 & 0.904 & & & 0.909 & & \\
\hline Q2 & 0.583 & 1.12 & $(0.74,1.69)$ & 0.618 & 1.11 & $(0.73,1.68)$ \\
\hline Q3 & 0.490 & 1.15 & $(0.76,1.75)$ & 0.707 & 1.08 & $(0.71,1.64)$ \\
\hline Q4 & 0.792 & 1.05 & $(0.7,1.59)$ & 0.884 & 0.97 & $(0.64,1.46)$ \\
\hline
\end{tabular}

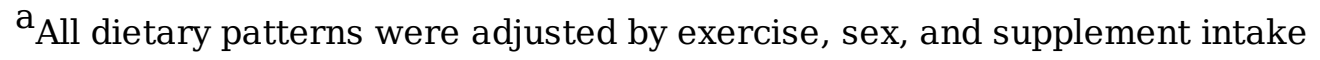

\section{Discussion}

By conducting a cross-sectional survey of 1246 people, we established a model to determine three dietary patterns in the dietary habits of the elderly aged 60 and above in Gongcheng County: balanced new dietary habits (vegetable and fruit-based dietary patterns), and traditional dietary habits (cereal and potato and oil tea-type diet model). The results of the three dietary patterns after adjustment of potential influencing variables, such as gender, exercise, and nutrition, did not change significantly. The dietary intake of the cereal and potato diet model and the oil tea diet model had no obvious relationship with cognitive function and did not affect cognitive ability. The vegetable and fruit-based diet model before and after the adjustment of nutritional factors showed that the model had a significant correlation with cognitive dysfunction and can reduce the risk of cognitive decline. This result suggested that when the dietary pattern was adjusted, the diet structure tended to be balanced, and the combination of nutrition and adherence to the vegetable and fruit-based diet may be a protective factor for cognitive function and slow down decline in cognitive function.

Traditional dietary pattern: the oil-tea-type diet model is characterized by the large intake of oil-tea and insufficient intake of vegetables, fruits, meat, cereals and potatoes. Table 3 shows that except the oil tea group, the factor load after the rotation of a group was less than 0.1 . Unhealthy eating patterns usually contain saturated/trans-unsaturated fatty acids, sugars, and rich calories are significantly correlated with cognitive decline in the elderly[39-46]. The Western diet consists mainly of saturated fatty acids and refined carbohydrates, which may increase the prevalence of Alzheimer's disease[47-50]. A study in Taiwan pointed out that the "meat" eating pattern is associated with increased risk of decreased fluency in language expression[51]. In Gongcheng County, the dietary pattern of cereals and potatoes is mainly 
characterized by rice intake. Dietary habits based on refined rice, meat, sweet drinks, and noodles[52] and dietary patterns based on rice intake may increase the risk of dyslipidemia[53]. A starch-rich diet reduces the cognitive ability of the elderly in China[54], especially among the carriers of apolipoprotein Eع4. Nutrients in dairy products[55] are commonly found in beans, fried food, and processed meat. The intake of food and peas accelerates cognitive decline in the elderly[56], and vitamins $E$ and $C$ as supplements can prevent vascular dementia[57]. Vitamin D[58] intake can alleviate cognitive decline[59,60], and adequate intake of antioxidant-rich fruits and vegetables has been shown to be closely related to damage in people with Alzheimer's disease[61,62]. Therefore, in a diet based on rice intake, insufficient intake of antioxidants and vitamins may explain the high risk of unbalanced diet and may increase the risk of cognitive impairment[63]. The traditional eating habits in the Guangxi region are less conducive to the prevention of decline in cognitive function than eating habits involving fruits and vegetables. Oil tea based dietary patterns based on oil tea and rice-based cereal-potato dietary patterns are not conducive to the maintenance of cognitive function. Nutrients derived from plants and animals are related to the retention of cognitive function. Compared with a new type of vegetable and fruit diet, diet lacking plantand animal-derived nutrients has lower antioxidant and anti-inflammatory effects[64], insufficient amount folic acid[65], and insufficient vascular secretions and high-quality proteins[66], leading to neuropathy, particularly in older adults[67]. Antioxidants in the brain can protect brain tissues from free radical damage[65]. Adherene to the Mediterranean diet and similar dietary patterns may exert great influence on vascular secretions and structural integrity of neuronal cell membranes, reduce oxidative stress, and enhance anti-inflammatory and antioxidant abilities[68,69]. Good protective effects[66] and tyrosine, catecholamine neurotransmitter, dopamine, norepinephrine, and epinephrine precursors are important components of protein food, and isoflavones and magnesium reduce oxidative stress and increase antiinflammatory properties related to high antioxidant capacity[70,71]. Reduction in dopamine level in a normal aging brain causes cognitive impairment[72]. Therefore, the correlation between dietary pattern and cognitive function is related to the combination of different nutrients and different dietary patterns. The potential impact between dietary patterns and cognitive impairment may have multidimensional biological effects.

When the dietary structure was adjusted, the intake of rice and food that is rich in starch but lacks antioxidants decreased, and the intake of fruits, vegetables, dairy products, fish, and other food groups increased. Remodeling resulted in a balanced diet that had a protective effect on cognitive function and delayed decline in cognitive function. As is well known, adherence to a diet involving high intake of vegetables and fish has protective effects[2]. In France, the dietary patterns of middle-aged people include fruits, whole grains, fresh dairy products, and vegetables[73]. Elderly men mostly eat fish, whereas elderly women mostly eat fruits and vegetables[74]. Loef[75] and Walach[76] confirmed that the nutritional combination of "Alzheimer's disease protection" is related to the high intake of fresh fruits and vegetables, whole grains, fish[77], and low-fat dairy products. In Finland, Italy, and the Netherlands, a healthy diet may be associated with good cognitive function in older men[78]. Strict adherence to the Mediterranean diet improves cognitive status[79-81]. A balanced diet is important for $\mathrm{MCl}$. The risk of illness is protective[82]. A long-term observation guided by a high-quality diet pattern showed improved cognitive function[83]. 
Multidomain interventions, including diet, may improve or help maintain the cognitive function of the elderly[84]. These studies are consistent with our research with regard to the protective effect of the vegetable and fruit diet model on cognitive function, reflecting that eating habit is a potentially modifiable factor that prevents multifactor cognitive impairment. The research results may guide the prevention and alleviation of cognitive decline in the elderly and provide a low-cost and feasible scientific method.

\section{Conclusions}

A cross-sectional study on the relationship between dietary patterns and cognitive functions of elderly people aged 60 and above in Gongcheng County, Guangxi was performed. According to the modeling results, traditional dietary models (grain and potato and oil tea-based dietary models) have no protective effects on cognitive function. When the diet structure was adjusted, a new balanced dietary pattern (vegetable and fruit-based dietary pattern) was found. Moreover, the model was modeled again, suggesting that the dietary pattern may have a protective effect on cognitive function and reflect eating habits. The importance of potentially modifiable factors to the prevention of multifactorial cognitive impairment is highlighted.

\section{Declarations}

Author Contributions: Ruoyu Gou, Writing - original draft, Writing - review \&editing. You Li, Writing - original draft, Writing - review \&editing. Weiyi Pang, Investigation, Data curation. Chunbao Mo, Investigation, Data curation. Jiansheng Cai, Investigation, Data curation. Tingyu Luo, Investigation. Tingyu Mai, Investigation. Dechan Tan, Methodology, Investigation. Kailian He, Investigation. Song Xiao, Investigation. Xu Tang, Investigation. Hongnan Li, Investigation. Zhiyong Zhang, Supervision, Project administration. Jian Qin, Conceptualization.

Funding: This study was supported by the National Natural Science Foundation of China (grant No. 81760577ه81960583 and 81560523), the Guangxi Science and Technology Development Project (grant No. AD17129003 and AD18050005), the Guangxi Natural Science Found for Innovation Research Team (2019GXNSFGA245002), and the Guangxi Scholarship Fund of Guangxi Education Department of China , Guangxi Graduate Education Innovation Project, Grant/Award Number: GYYK2021001 .

Institutional Review Board Statement: The ethics was reviewed and approved by the ethics committee of the School of Medicine of Guilin Medical College, and informed consent forms were signed for the participation system $₫$ No:20180702-3 and July 2,2018囚

Informed Consent Statement: Informed consent was obtained from all subjects involved in the study.

Data Availability Statement: The data presented in this study are available on request from the corresponding author. 
Acknowledgments: The authors would like to acknowledge all participants in the study for their time and invaluable contributions.

Declaration of competing interest :The authors declare that they have no known competing financial interests or personal relationships that could have appeared to influence the work reported in this paper.

\section{References}

1. Deary, I.J.; Corley, J.; Gow, A.J.; Harris, S.E.; Houlihan, L.M.; Marioni, R.E.; Penke, L.; Rafnsson, S.B.; Starr, J.M. Age-associated cognitive decline. Br Med Bull 2009, 92, 135-152, doi:10.1093/bmb/ldp033.

2. Ma, W.; Hagan, K.A.; Heianza, Y.; Sun, Q.; Rimm, E.B.; Qi, L. Adult height, dietary patterns, and healthy aging. American Journal of Clinical Nutrition 2017, 589-708.

3. Cheung, B.H.; Ho, I.C.; Chan, R.S.; Sea, M.M.; Woo, J. Current evidence on dietary pattern and cognitive function. Adv Food Nutr Res 2014, 71, 137-163, doi:10.1016/B978-0-12-800270-4.00004-3.

4. Martin Prince, A.W., Maëlenn Guerchet,Gemma-Claire Ali. WorldAlzheimerReport2015.pdf>. Alzheimer's Disease International (ADI) August 2015, 87.

5. Pang, S.J.; Jia, S.S.; Man, Q.Q.; Song, S.; Li, Y.Q.; Song, P.K.; Zhao, W.H.; Zhang, J. Dietary Cholesterol in the Elderly Chinese Population: An Analysis of CNHS 2010-2012. Nutrients 2017, 9, $934-$.

6. Jia, L.; Du, Y.; Chu, L.; Zhang, Z.; Qiu, Q. Prevalence, risk factors, and management of dementia and mild cognitive impairment in adults aged 60 years or older in China: a cross-sectional study. The Lancet Public Health 2020, 5, e661-e671.

7. Sosa-Ortiz, A.L.; Acosta-Castillo, I.; Prince, M.J. Epidemiology of dementias and Alzheimer's disease. Arch Med Res 2012, 43, 600-608, doi:10.1016/j.arcmed.2012.11.003.

8. Lane, C.A.; Hardy, J.; Schott, J.M. Alzheimer's disease. European Journal of Neurology 2017, 25.

9. Minhas, P.S.; Latif-Hernandez, A.; Mcreynolds, M.R.; Durairaj, A.S.; Andreasson, K.I. Restoring metabolism of myeloid cells reverses cognitive decline in ageing. Nature 2021.

10. Jun-Peng Zhuang, G.W., Qi Cheng,Li-Ling Wang. Cognitive impairment and the associated riskfactors among the elderly in the Shanghai urbanarea_ a pilot study from China.pdf $>$. Translational Neurodegeneration 2012.

11. Luchsinger, J.A.; Mayeux, R. Dietary factors and Alzheimer's disease. The Lancet Neurology 2004, 3, 579-587, doi:10.1016/s1474-4422(04)00878-6.

12. Solfrizzi, V.; ustodero, C.C.; Lozupone, M.; Imbimbo, B.P.; Valiani, V.; Agosti, P.; Schilardi, A.; D'Introno, A.; Montagna, M.L.; Calvani, M. Relationships of Dietary Patterns, Foods, and Micro- and Macronutrients 
with Alzheimer's Disease and Late-Life Cognitive Disorders: A Systematic Review. Journal of Alzheimerl"s Disease 2017, 59, 815-849.

13. Attuquayefio, T.; Stevenson, R.J. A systematic review of longer-term dietary interventions on human cognitive function: Emerging patterns and future directions. Appetite 2015, 95, 554-570.

14. Martha Clare Morris, D.A.E., Christine C. Tangney. Fish Consumption and Cognitive Decline With Agein a Large Community Study.pdf>. Arch Neurol 2005.

15. Joseph, J.; Cole, G.; Head, E.; Ingram, D. Nutrition, brain aging, and neurodegeneration. J Neurosci 2009, 29, 12795-12801, doi:10.1523/JNEUROSCI.3520-09.2009.

16. Kulzow, N.; Witte, A.V.; Kerti, L.; Grittner, U.; Schuchardt, J.P.; Hahn, A.; Floel, A. Impact of Omega-3 Fatty Acid Supplementation on Memory Functions in Healthy Older Adults. J Alzheimers Dis 2016, 51, 713-725, doi:10.3233/JAD-150886.

17. S. Kalmijn, E.J.M.F., L J. Launer,D. Kromhout. Polyunsaturated Fatty Acids, Antioxidants, and Cognitive Function in Very.pdf>. American Journal of Epidemiology 1997.

18. Yian Gu, J.W.N., Yaakov Stern. Food Combination and Alzheimer Disease RiskA Protective Diet.pdf>. Arch Neurol 2010.

19. Wang; Cui-Jiang; Yang; Tong-Fang; Guang-Sheng; Zhao; Yuan-Yuan; Li-Jun; Bi; Bin-Na. Association between dietary patterns and depressive symptoms among middle-aged adults in China in 2016-2017. PSYCHIATRY RESEARCH 2018.

20. Petersson, S.D.; Philippou, E. Mediterranean Diet, Cognitive Function, and Dementia: A Systematic Review of the Evidence. Adv Nutr 2016, 7, 889-904, doi:10.3945/an.116.012138.

21. Aridi, Y.S.; Walker, J.L.; Wright, O.R.L. The Association between the Mediterranean Dietary Pattern and Cognitive Health: A Systematic Review. Nutrients 2017, 9, doi:10.3390/nu9070674.

22. Catherine Fe'art, C.c.S., Virginie Rondeau. Adherence to a Mediterranean Diet,Cognitive Decline, and Risk of Dementia.pdf>. JAMA 2009.

23. Duplantier, S.C.; Gardner, C.D. A Critical Review of the Study of Neuroprotective Diets to Reduce Cognitive Decline. Nutrients 2021, 13, doi:10.3390/nu13072264.

24. Theodora; Psaltopoulou; Theodoros; N.; Sergentanis; Demosthenes; B.; Panagiotakos; loannis; N. Mediterranean diet, stroke, cognitive impairment, and depression: A meta-analysis. Annals of Neurology 2013, 74, 580-591.

25. Morris, M.C.; Tangney, C.C.; Wang, Y.; Sacks, F.M.; Bennett, D.A.; Aggarwal, N.T. MIND diet associated with reduced incidence of Alzheimer's disease. Alzheimers Dement 2015, 11, 1007-1014, 
doi:10.1016/j.jalz.2014.11.009.

26. Morris, M.C.; Tangney, C.C.; Wang, Y.; Sacks, F.M.; Barnes, L.L.; Bennett, D.A.; Aggarwal, N.T. MIND diet slows cognitive decline with aging. Alzheimers Dement 2015, 11, 1015-1022, doi:10.1016/j.jalz.2015.04.011.

27. Zheng, G.; Sayama, K.; Okubo, T.; Juneja, L.R.; Oguni, I. Anti-obesity effects of three major components of green tea, catechins, caffeine and theanine, in mice. In vivo (Athens, Greece) 2004, 18, 5562.

28. Kyoung; Yun; Kim; Jung-Mi; Yun. Association between diets and mild cognitive impairment in adults aged 50 years or older. Nutrition Research \& Practice 2018.

29. Park, J.H.; Park, Y.N.; Ko, H.J.J.I.J.o.G.P. Modification of the mini-mental state examination for use with the elderly in a non-western society. Part II: Cutoff points and their diagnostic validities. 1991, 6, 875-882.

30. Petersen, R.C.; Smith, G.E.; Waring, S.C.; Ivnik, R.J.; Tangalos, E.G.; Kokmen, E. Mild Cognitive Impairment. Archives of Neurology 1999, 56, 303.

31. Liu, X.; Wang, X.; Lin, S.; Song, Q.; Lao, X.; Yu, I.T. Reproducibility and Validity of a Food Frequency Questionnaire for Assessing Dietary Consumption via the Dietary Pattern Method in a Chinese Rural Population. PLoS One 2015, 10, e0134627, doi:10.1371/journal.pone.0134627.

32. security, C.C.f.D.C.a.P.n.a.f. Chinese Food Composition Table; Chinese Food Composition Table: 2009.

33. JI Linong, R.X.M.Y.e.a. Chinese expert recommendations on basal insulin treatment in adult type2diabetes mellitus. Chin J Diabetes October 2020, 28, 10, doi:10.3969/j.issn.1006-6187.2020.10.001.

34. (China), H.A. Chinese Guidelines for Prevention and Treatment of Hypertension 2018 revised edition. PreventionandTreatmentofCardio_Cerebral_VascularDisease Jan2018, 19, 44.

35. Tao Guoshu, Z.H. 2016 Chinese guideline for the management of dyslipidemia in adults. ChinJ Health Care Med August 2017, 19, 3, doi:10.3969/囚issn.1674-3245.2017.04.029.

36. Kim, K.Y.; Yun, J.M. Association between diets and mild cognitive impairment in adults aged 50 years or older. Nutr Res Pract 2018, 12, 415-425, doi:10.4162/nrp.2018.12.5.415.

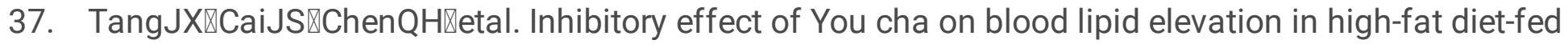

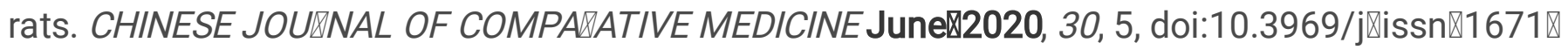
7856.2020.06.014. 
38. Schulze, M.B.; Hoffmann, K.; Kroke, A.; Boeing, H.J.B.J.o.N. An approach to construct simplified measures of dietary patterns from exploratory factor analysis. 2003, 89, 409-418.

39. Morris, M.C.; Evans, D.; Bienias, J.L.; Tangney, C.C.; Wilson, R.S.J.N. Dietary fat intake and 6-year cognitive change in an older biracial community population. 2004, 62, 1573-1579.

40. Luchsinger, J.A.; Tang, M.X.; Sh Ea , S.; Mayeux, R.J.A.o.N. Caloric Intake and the Risk of Alzheimer Disease. 2002, 59, 1258.

41. Laitinen, M.H.; Ngandu, T.; Rovio, S.; Helkala, E.L.; Kivipelto, M.J.D.; Disorders, G.C. Fat Intake at Midlife and Risk of Dementia and Alzheimer's Disease: A Population-Based Study. 2006, 22, 99-107.

42. Almeida, O.P.; Norman, P.; Hankey, G.; Jamrozik, K.; Flicker, L.J.A.J.o.G.P. Successful Mental Health Aging: Results From a Longitudinal Study of Older Australian Men. 2006, 14, 27-35.

43. Eskelinen, M.H.; Ngandu, T.; Helkala, E.L.; Tuomilehto, J.; Kivipelto, M.J.A.; Dementia. Fat intake at midlife and cognitive impairment later in life: a population-based CAIDE study. 2010, 23, 741-747.

44. K. Mehlig, I.S., X. Guo, M. Schütze, D. Gustafson, M. Waern, S. stling, C. Bjrkelund; Epidemiology, L.L.J.A.J.o. Alcoholic beverages and incidence of dementia: 34-year follow-up of the prospective population study of women in Goteborg. 2008, 167, 684-691.

45. Kalmijn, S.; Launer, L.J.; Ott, A.; Witteman, J.C.; Hofman, A.; Breteler, M.M.J.A.o.N. Dietary fat intake and the risk of incident dementia in the Rotterdam Study. 2010, 42, 776-782.

46. Vincenzo; Solfrizzi; and; Alessia; D'Introno; and; Anna; M.; Colacicco; Gerontology, a.J.E. Dietary fatty acids intake: possible role in cognitive decline and dementia - ScienceDirect. 2005, 40, 257-270.

47. Berrino, F. [Western diet and Alzheimer's disease]. Epidemiologia E Prevenzione 2002, 26, 107.

48. Grant, W.B.; Campbell, A.; Itzhaki, R.F.; Savory, J. The significance of environmental factors in the etiology of Alzheimer's disease. Journal of Alzheimerl"s Disease 2002, 4, 179-189.

49. Pasinetti, G.M.; Eberstein, J.A. Metabolic syndrome and the role of dietary lifestyles in Alzheimer's disease. J Neurochem 2008, 106, 1503-1514, doi:10.1111/j.1471-4159.2008.05454.x.

50. Hu, F.B.; Liu, S.; Dam, R.V. Diet and risk of Type II diabetes: the role of types of fat and carbohydrate. Diabetologia 2001, 44, 805-817.

51. Chen, Y.C.; Jung, C.C.; Chen, J.H.; Chiou, J.M.; Lee, M.S.J.J.o.t.A.G.S. Association of Dietary Patterns With Global and Domain-Specific Cognitive Decline in Chinese Elderly. 2017, 65, 1159.

52. Baik, I.; Lee, M.; Jun, N.R.; Lee, J.Y.; Shin, C. A healthy dietary pattern consisting of a variety of food choices is inversely associated with the development of metabolic syndrome. Nutr Res Pract 2013, 7, 233241, doi:10.4162/nrp.2013.7.3.233.

Page 21/24 
53. Song, S.J.; Lee, J.E.; Paik, H.Y.; Park, M.S.; Song, Y.J. Dietary patterns based on carbohydrate nutrition are associated with the risk for diabetes and dyslipidemia. Nutr Res Pract 2012, 6, 349-356, doi:10.4162/nrp.2012.6.4.349.

54. Xu, X.; Parker, D.; Shi, Z.; Byles, J.; Hall, J.; Hickman, L. Dietary Pattern, Hypertension and Cognitive Function in an Older Population: 10-Year Longitudinal Survey. Front Public Health 2018, 6, 201, doi:10.3389/fpubh.2018.00201.

55. Federica; Prinelli; Laura; Fratiglioni; Massimo; Musicco; Ingegerd; Johansson; Fulvio; nutrition, A.J.C. The impact of nutrient-based dietary patterns on cognitive decline in older adults. 2018.

56. Mio; Ozawa; Martin; Shipley; Mika; Kivimaki; Archana; Singh-Manoux; Eric; J. Dietary pattern, inflammation and cognitive decline: The Whitehall II prospective cohort study. Clinical Nutrition 2017.

57. D A, O. Association of vitamin $\mathrm{E}$ and $\mathrm{C}$ supplement use with cognitive function and dementia in elderly men. Neurology 2000, 6.

58. Pettersen, J.A. Does high dose vitamin D supplementation enhance cognition?: A randomized trial in healthy adults. Exp Geronto/ 2017, 90, 90-97, doi:10.1016/j.exger.2017.01.019.

59. Devore, E.E.; Kang, J.H.; Breteler, M.M.; Grodstein, F. Dietary intakes of berries and flavonoids in relation to cognitive decline. Ann Neuro/ 2012, 72, 135-143, doi:10.1002/ana.23594.

60. McNeill, G.; Winter, J.; Jia, X. Diet and cognitive function in later life: a challenge for nutrition epidemiology. Eur J Clin Nutr 2009, 63 Supp/ 1, S33-37, doi:10.1038/ejcn.2008.62.

61. Lourida, I.A.; Soni, M.B.; Thompsoncoon, J.A.; Purandare, N.A.; Lang, I.; Ukoumunne, O.; Llewellyn, D. Mediterranean Diet, Cognitive Function, and Dementia: A Systematic Review. [Miscellaneous Article]. 2013.

62. Marianne J Engelhart1, M.I.G., Annemieke Ruitenberg,John C van Swieten,Albert Hofman,Jacqueline C M Witteman,Monique M B Breteler. Dietary Intake of Antioxidantsand Risk of Alzheimer Disease. JAMA 2002, 10.1001/jama.287.24.3223, doi:10.1001/jama.287.24.3223.

63. Kim, J.; Yu, A.; Bo, Y.C.; Nam, J.H.; Yang, Y.J.J.E.J.o.N. Dietary patterns and cognitive function in Korean older adults. 2014, 54, 309-318.

64. Rubio-Perez, J.M.; Morillas-Ruiz, J.M. A review: inflammatory process in Alzheimer's disease, role of cytokines. ScientificWorldJournal 2012, 2012, 756357, doi:10.1100/2012/756357.

65. Herrmann, W.; Obeid, R. Homocysteine: a biomarker in neurodegenerative diseases. Clinical Chemistry \& Laboratory Medicine 2011, 49, 435-441. 
66. Scarmeas, N. Mediterranean diet improves cognition:the PREDIMED-NAVARRA randomised trial. $J$ Neurol Neurosurg Psychiatry 2013, 84, 1297, doi:10.1136/jnnp-2013-305153.

67. Gandhi, S.; Abramov, A.Y. Mechanism of oxidative stress in neurodegeneration. Oxid Med Cell Longev 2012, 2012, 428010, doi:10.1155/2012/428010.

68. Panza, F.; Frisardi, V.; Seripa, D.; Logroscino, G.; Imbimbo, B.P.; Pilotto, A.; Solfrizzi, V. Diet and Alzheimer's disease risk factors or prevention: the current evidence. Expert Review of Neurotherapeutics 2011, 11, 677.

69. Samieri, C.; Grodstein, F.; Rosner, B.A.; Kang, J.H.; Cook, N.R.; Manson, J.E.; Buring, J.E.; Willett, W.C.; Okereke, O.I. Mediterranean diet and cognitive function in older age. Epidemiology 2013, 24, 490-499, doi:10.1097/EDE.0b013e318294a065.

70. Hughes, T.F.; Becker, J.T.; Lee, C.W.; Chang, C.H.; Ganguli, M.; Pelletier, A.; Barul, C.; Féart, C.; Helmer, C.; Bernard, C. Brain foods: the effects of nutrients on brain function. 2015.

71. Guyonnet, S.G.; Abellan, V.; Andrieu, S.; Gateau, P.B.; Berr, C.; Bonnefoy, M.; Dartigues, J.F.; Groot, L.D.; Ferry, M.; Galan, P. IANA task force on nutrition and cognitive decline with aging. Journal of Nutrition Health \& Aging 2007, 11, 132.

72. Young; V., R. Amino acids and proteins in relation to the nutrition of elderly people. Age \& Ageing 1990, 19, 10-24.

73. Kesse-Guyot, E.; Andr Ee Va, V.A.; Jeandel, C.; Ferry, M.; Hercberg, S.; Galan, P.J.J.o.N. A Healthy Dietary Pattern at Midlife Is Associated with Subsequent Cognitive Performance1,2,3. 2012.

74. Samieri, C.; Jutand, M.A.; Féart, C.; Capuron, L.; Letenneur, L.; Barberger-Gateau, P.J.J.o.t.A.D.A. Dietary Patterns Derived by Hybrid Clustering Method in Older People: Association with Cognition, Mood, and Self-Rated Health. 2008, 108, 1461-1471.

75. Ye; X.; Bhupathiraju; S., N.; Tucker; K., L.J.B.J.o.N. Variety in fruit and vegetable intake and cognitive function in middle-aged and older Puerto Rican adults. 2013.

76. Loef, M.; Walach, H.J.J.o.N.H.; Aging. Fruit, vegetables and prevention of cognitive decline or dementia: A systematic review of cohort studies. 2012, 16, 626.

77. Kalmijn, S.; Launer, L.J.; Ott, A.; Witteman, J.C.; Hofman, A.; Breteler, M.M. Dietary fat intake and the risk of incident dementia in the Rotterdam Study. Annals of Neurology 2010, 42, 776-782.

78. Huijbregts; P.; P.; C.; W.; eskens, F.; E.; J.; Nutrition, M.J.E.J.o.C. Dietary patterns and cognitive function in elderly men in Finland, Italy and the Netherlands. 1998. 
79. Amicis, R.D.; Leone, A.; Foppiani, A.; Osio, D.; Lewandowski, L.; Giustizieri, V.; Cornelio, P.; Cornelio, F.; Imperatori, S.F.; Cappa, S.F.J.J.o.t.A.C.o.N. Mediterranean Diet and Cognitive Status in Free-Living Elderly: A Cross-Sectional Study in Northern Italy. 2018, 1.

80. Scarmeas, N.; Stern, Y.; Mayeux, R.; Manly, J.J.; Schupf, N.; Luchsinger, J.A. Mediterranean Diet and Mild Cognitive Impairment. Archives of Neurology 2009, 66, 216-225.

81. Trichopoulou, A.; Kyrozis, A.; Rossi, M.; Katsoulis, M.; Trichopoulos, D.; Vecchia, C.L.; Lagiou, P. Mediterranean diet and cognitive decline over time in an elderly Mediterranean population. European Journal of Nutrition 2015.

82. Zhou, H. Study on cognitive function and diet of the aged in rural area of Jiaozhou City. Qingdao University, 2020.

83. Zhu, N.; Jacobs, D.R.; Meyer, K.A.; He, K.; Launer, L.; Reis, J.P.; Yaffe, K.; Sidney, S.; Whitmer, R.A.; Steffen, L.M. COGNITIVE FUNCTION IN A MIDDLE AGED COHORT IS RELATED TO HIGHER QUALITY DIETARY PATTERN 5 AND 25 YEARS EARLIER: THE CARDIA STUDY. other 2015, 19.

84. Ngandu, T.; Lehtisalo, J.; Solomon, A.; Levälahti, E.; Ahtiluoto, S.; Antikainen, R.; Bäckman, L.; Hänninen, T.; Jula, A.; Laatikainen, T.J.T.L. A 2 year multidomain intervention of diet, exercise, cognitive training, and vascular risk monitoring versus control to prevent cognitive decline in at-risk elderly people (FINGER): a randomised controlled trial. 2015.

\section{Supplementary Files}

This is a list of supplementary files associated with this preprint. Click to download.

- Table4.png

- Table6.png 